\title{
Supplementary Appendix for:
}

\section{"Robust Inference for Inverse Stochastic Dominance"}

\author{
Francesco Andreoli
}

Luxembourg Institute of Socio-Economic Research (LISER), 11 Porte des Sciences, L-4366

Esch-sur-Alzette / Belval Campus, Luxembourg. E-mail: francesco.andreoli@liser.lu.

\begin{abstract}
This document reports the proofs of Lemma 1 and Remark 2 in the article. It also reports an extended account of the results of a Monte Carlo study. The simulation study allows to verify the behavior of the influence function estimator and of the bootstrap estimator for inverse stochastic dominance when the sample size and the number of abscissae are exogenously manipulated. The study also compares the performances of the ISD tests with standard stochastic dominance consistent and potentially inconsistent tests. Simulations of size and powers of the tests are derived in a baseline setting (also discussed in the article) and in a case where data contamination is artificially introduced. Simulated distributions are taken from empirical models of earnings and durable consumption dynamics in the U.S.
\end{abstract}




\section{A Proofs of results presented in the article}

\section{A.1 Proof of Lemma 1}

Lemma 1 If the domain of $F$ is bounded from below then $\phi_{i}\left(p, \widehat{\Lambda}_{k}\right)$ is bounded.

Proof. Note that an abscissa $p=F_{\varepsilon}^{(i)}(y)$ is related to an ordinate $F(y)$ in the population of interest by the following relation:

$$
F(y)=\frac{F_{\varepsilon}^{(i)}(y)-\varepsilon \mathbf{1}\left(y \geq y_{i}\right)}{1-\varepsilon} .
$$

The following equivalence thus holds:

$$
\begin{aligned}
\widehat{\Lambda}_{k}^{F_{\varepsilon}^{(i)}}(p) & =\frac{1}{(k-3) !} \int_{0}^{p}(p-t)^{k-3} \widehat{G L}_{F_{\varepsilon}^{(i)}}(t) d t \\
& =\frac{1}{(k-3) !} \int_{0}^{p}\left(p-\frac{t-\varepsilon \mathbf{1}\left(y \geq y_{i}\right)}{1-\varepsilon}\right)^{k-3} \widehat{G L}\left(\frac{t-\varepsilon \mathbf{1}\left(y \geq y_{i}\right)}{1-\varepsilon}\right) d t
\end{aligned}
$$

Letting $t=F(y)$ and making use of the algebra of Hadamard differentiation (see, for instance, Deville 1999), one obtains that:

$$
\begin{aligned}
\phi_{i}\left(p, \widehat{\Lambda}_{k}\right)= & \left.\frac{1}{(k-3) !} \int_{0}^{p} \frac{d}{d \varepsilon}\left(p-\frac{t-\varepsilon \mathbf{1}\left(F^{-1}(t) \geq y_{i}\right)}{1-\varepsilon}\right){ }^{k-3} \widehat{G L}\left(\frac{t-\varepsilon \mathbf{1}\left(F^{-1}(t) \geq y_{i}\right)}{1-\varepsilon}\right)\right|_{\varepsilon \rightarrow 0} d t \\
= & \frac{1}{(k-3) !} \int_{0}^{p}(k-3)(p-t)^{k-4}\left(\mathbf{1}\left(F^{-1}(t) \geq y_{i}\right)-t\right) \widehat{G L}(t)+ \\
& +\left.(p-t)^{k-3} \frac{d}{d \varepsilon} \widehat{G L}\left(\frac{t-\varepsilon \mathbf{1}\left(F^{-1}(t) \geq y_{i}\right)}{1-\varepsilon}\right)\right|_{\varepsilon \rightarrow 0} d t \\
= & \frac{1}{(k-3) !} \int_{0}^{p}(k-3)(p-t)^{k-4}\left(\mathbf{1}\left(F^{-1}(t) \geq y_{i}\right)-t\right) \widehat{G L}(t)+ \\
& +(p-t)^{k-3}\left(p F^{-1}(t)-\widehat{G L}(t)+\mathbf{1}\left(F^{-1}(t) \geq y_{i}\right)\left(y_{i}-F^{-1}(t)\right)\right) d t .
\end{aligned}
$$

The last line holds when the derivatives are evaluated at $\varepsilon=0$, in which case the influence function of $\widehat{G L}$ is provided in Cowell and Victoria-Feser (2002). Integration by parts, and the fact that $\int_{0}^{p} \mathbf{1}\left(F^{-1}(t) \geq y_{i}\right) h(t) d t=\mathbf{1}\left(F^{-1}(p) \geq y_{i}\right) \int_{0}^{p} h(t) d t$ for any 
function $h$, give that

$$
\phi_{i}\left(p, \widehat{\Lambda}_{k}\right)=\mathbf{1}\left(F^{-1}(p) \geq y_{i}\right) y_{i} \frac{p^{k-2}}{(k-2) !}
$$

which is bounded if $F$ is bonded from below.

\section{A.2 Proof of Remark 2}

Remark 2 Suppose that for a set of population abscissae $\left\{p_{j} \mid j=1, \ldots m\right\}$, the distribution function $F$ is continuous and derivable in $p_{j}$ with density function $f\left(F^{-1}\left(p_{j}\right)\right)>$ 0 for every $j$. Then, for $p_{j} \geq p_{j^{\prime}}, \sigma_{1}\left(p_{j}, p_{j^{\prime}}\right)$ and $\sigma_{2}\left(p_{j}, p_{j^{\prime}}\right)$ coincide with the covariance estimators in Beach and Davidson (1983), Lemma 1 and Theorem 1 respectively.

Proof. I first show that when $p \leq p^{\prime}$ (as it is the case for $p_{j} \leq p_{j^{\prime}}$ ), it holds that

$$
\sigma_{1}\left(p, p^{\prime}\right)=\mathrm{E}\left[\phi\left(p, F^{-1}\right) \phi\left(p^{\prime}, F^{-1}\right)\right]=\frac{p\left(1-p^{\prime}\right)}{f\left(F^{-1}(p)\right) f\left(F^{-1}\left(p^{\prime}\right)\right)},
$$

where the "hat" symbol delimiting the empirical processes is dropped in the proof to avoid cumbersome notation. I also drop references to the observations, $i$, so that the influence function is expressed conditional on a generic income realization $y$. The formula above denotes the quantiles covariance in Beach and Davidson (1983), Lemma 1. To verify this equation, I make use of the definition of the influence function of a quantile of $F$, denoted

$$
\phi\left(p, F^{-1}\right)=\left(p-\mathbf{1}\left(F^{-1}(p) \geq y\right)\right) / f\left(F^{-1}(p)\right)
$$

Substituting into the definition of the IF estimator for quantiles and solving the expectation, one has

$$
\begin{aligned}
\sigma_{1}\left(p, p^{\prime}\right) & =\mathrm{E}\left[\frac{p-\mathbf{1}\left(F^{-1}(p) \geq y\right)}{f\left(F^{-1}(p)\right)} \frac{p^{\prime}-\mathbf{1}\left(F^{-1}\left(p^{\prime}\right) \geq y\right)}{f\left(F^{-1}\left(p^{\prime}\right)\right)}\right] \\
& =\frac{p p^{\prime}-p \mathrm{E}\left[\mathbf{1}\left(F^{-1}\left(p^{\prime}\right) \geq y\right)\right]-p^{\prime} \mathrm{E}\left[\mathbf{1}\left(F^{-1}(p) \geq y\right)\right]+\mathrm{E}\left[\mathbf{1}\left(F^{-1}(p) \geq y\right) \mathbf{1}\left(F^{-1}\left(p^{\prime}\right) \geq y\right)\right]}{f\left(F^{-1}(p)\right) f\left(F^{-1}\left(p^{\prime}\right)\right)} .
\end{aligned}
$$


The result follows by simplifying the expression, making use of the fact that the expectation of a step function is $\mathrm{E}\left[\mathbf{1}\left(F^{-1}(p) \geq y\right)\right]=\int_{0}^{y} d F(y)=p$ whenever evaluated at $F(y)=p$.

The second result that I prove is that

$$
\begin{aligned}
\sigma_{2}\left(p, p^{\prime}\right) & =\mathrm{E}\left[\phi_{i}(p, G L) \phi_{i}\left(p^{\prime}, G L\right)\right] \\
& =p\left[\lambda^{2}+\left(1-p^{\prime}\right)\left(F^{-1}(p)-\gamma\right)\left(F^{-1}\left(p^{\prime}\right)-\gamma^{\prime}\right)+\left(F^{-1}(p)-\gamma\right)\left(\gamma^{\prime}-\gamma\right)\right]
\end{aligned}
$$

where $\gamma$ is the ratio between the incomplete moments of order one and zero of the distribution $F$ calculated at quantile $p$ (Butler and McDonald 1989), satisfying

$$
p \gamma:=G L(p)=\int_{0}^{F^{-1}(p)} y d F(y)
$$

while $\lambda^{2}$ is defined as the variance of a random variable distributed as $F$ conditional on $y \leq F^{-1}(p)$. It is implicitly given by the following:

$$
p\left(\lambda^{2}+\gamma^{2}\right)=\int_{0}^{F^{-1}(p)} y^{2} d F(y)
$$

The formula corresponds to the covariance between the intercepts of the generalized Lorenz curve estimator calculated at abscissae $\left(p, p^{\prime}\right)$ such that $p \leq p^{\prime}$, and denoted by $\omega$ in Beach and Davidson (1983), Theorem 1. To construct the proof, recall that

$$
\phi(p, G L)=p F^{-1}(p)-G L(p)+\mathbf{1}\left(y \leq F^{-1}(p)\right)\left(y-F^{-1}(p)\right) .
$$

Substituting for (1) into $\mathrm{E}\left[\phi(p, G L) \phi\left(p^{\prime}, G L\right)\right]$, one obtains an expectation of a linear function, which can be decomposed into the following elements

$$
\sigma_{2}\left(p, p^{\prime}\right)=\mathrm{E}_{1}+\mathrm{E}_{2}-\mathrm{E}_{3}+\mathrm{E}_{4}-\mathrm{E}_{5}+\mathrm{E}_{6},
$$


where

$$
\begin{aligned}
\mathrm{E}_{1} & :=\mathrm{E}\left[p F^{-1}(p) p^{\prime} F^{-1}\left(p^{\prime}\right)-G L(p) p^{\prime} F^{-1}\left(p^{\prime}\right)+G L(p) G L\left(p^{\prime}\right)\right] \\
& =p F^{-1}(p) p^{\prime} F^{-1}\left(p^{\prime}\right)-p \gamma p^{\prime} F^{-1}\left(p^{\prime}\right)+p \gamma p^{\prime} \gamma^{\prime} \\
\mathrm{E}_{2} & :=\mathrm{E}\left[\mathbf{1}\left(y \leq F^{-1}\left(p^{\prime}\right)\right)\left(y-F^{-1}\left(p^{\prime}\right)\right) p F^{-1}(p)\right] \\
& =p F^{-1}(p) \int_{0}^{F^{-1}\left(p^{\prime}\right)}\left(y-F^{-1}\left(p^{\prime}\right)\right) d F(y)=p F^{-1}(p) p^{\prime} \gamma^{\prime}-p F^{-1}(p) p^{\prime} F^{-1}\left(p^{\prime}\right) ; \\
\mathrm{E}_{3} & :=\mathrm{E}\left[G L(p) \mathbf{1}\left(y \leq F^{-1}\left(p^{\prime}\right)\right)\left(y-F^{-1}\left(p^{\prime}\right)\right)\right] \\
& =p \gamma \int_{0}^{F^{-1}\left(p^{\prime}\right)}\left(y-F^{-1}\left(p^{\prime}\right)\right) d F(y)=p \gamma p^{\prime} \gamma^{\prime}-p \gamma p^{\prime} F^{-1}\left(p^{\prime}\right) ; \\
\mathrm{E}_{4} & :=\mathrm{E}\left[\mathbf{1}\left(y \leq F^{-1}(p)\right)\left(y-F^{-1}(p)\right) p^{\prime} F^{-1}\left(p^{\prime}\right)\right] \\
& =p^{\prime} F^{-1}\left(p^{\prime}\right) \int_{0}^{F^{-1}(p)}\left(y-F^{-1}(p)\right) d F(y)=p^{\prime} F^{-1}\left(p^{\prime}\right) p \gamma-p^{\prime} F^{-1}\left(p^{\prime}\right) p F^{-1}(p) ; \\
\mathrm{E}_{5} & :=\mathrm{E}\left[\mathbf{1}\left(y \leq F^{-1}(p)\right)\left(y-F^{-1}(p)\right) G L\left(p^{\prime}\right)\right] \\
& =p^{\prime} \gamma^{\prime} \int_{0}^{F^{-1}(p)}\left(y-F^{-1}(p)\right) d F(y)=p^{\prime} \gamma^{\prime} p \gamma-p^{\prime} \gamma^{\prime} p F^{-1}(p) ; \\
\mathrm{E}_{6} & :=\mathrm{E}\left[\mathbf{1}\left(y \leq F^{-1}(p)\right) \mathbf{1}\left(y \leq F^{-1}\left(p^{\prime}\right)\right)\left(y-F^{-1}(p)\right)\left(y-F^{-1}\left(p^{\prime}\right)\right)\right] \\
& =\int_{0}^{F^{-1}(p)} y^{2} d F(y)+p F^{-1}(p) F^{-1}\left(p^{\prime}\right)-F^{-1}(p) p \gamma-F^{-1}\left(p^{\prime}\right) p \gamma \\
& =p\left(\lambda^{2}+\gamma^{2}\right)+p F^{-1}(p) F^{-1}\left(p^{\prime}\right)-F^{-1}(p) p \gamma-F^{-1}\left(p^{\prime}\right) p \gamma .
\end{aligned}
$$

The last line in $\mathrm{E}_{6}$ follows by the fact that $p \leq p^{\prime}$. Putting all together and simplifying, one obtains:

$\sigma_{2}\left(p, p^{\prime}\right)=p\left[\lambda^{2}+p^{\prime}\left(F^{-1}(p)-\gamma\right)\left(F^{-1}\left(p^{\prime}\right)-\gamma^{\prime}\right)+\gamma^{2}+F^{-1}(p) F^{-1}\left(p^{\prime}\right)-F^{-1}(p) \gamma-F^{-1}\left(p^{\prime}\right) \gamma\right]$

Adding and subtracting the quantities $F^{-1}(p) \gamma^{\prime}$ and $\gamma \gamma^{\prime}$ and rearranging, one obtains the desired result. 


\section{B Monte Carlo results, extended version}

The size and power properties of the estimators discussed so far are assessed through a series of Monte Carlo experiments. An account of the results presented in this session is reported in Section 5 of the main article. Each experiment involves tests for ISD at order one, two and three, the relevant case where standard stochastic dominance analysis and ISD analysis differ. The Monte Carlo experiment provides intuitions on the behavior of the different estimators when the sample size is relatively small, and allows to draw conclusions about the effect of increasing the sample size and manipulating the number of threshold at which ISD is assessed.

The estimators of ISD $k$ have been used to test the null hypothesis that a distribution $F$ dominates another distribution $G$ at some order $k$, that is:

$$
H_{0}^{k}: \quad F \succcurlyeq_{I S D k} G, \quad H_{1}^{k}: \text { non-dominance. }
$$

The Monte Carlo experiment consists in simulating 1000 independent sample draws from parametric models of $F$ and $G$. The design of each parametric model is inspired by Barrett and Donald (2009), whereby each simulated draw of a variable $Y_{i}$ at simulation stage $i$ is generated by a lognormal distribution, i.e. $Y_{i}=\exp \left(\sigma Z_{i}+\mu\right)$ where $Z_{i}$ is a realization of a $\mathcal{N}(0,1)$ random variable and $(\sigma, \mu)$ are the dispersion and location parameters. Each experiment involves the simulation of three samples of size 100, 500 and 1500 respectively. For each experiment, the null $H_{0}^{k}$ is tested for $k \in\{1,2,3\}$ (both in strict dominance and equality forms) using different estimators for the asymptotic covariances, while setting the number of abscissae to $m \in\{5,10,20\}$. These abscissae correspond to population proportions increments of respectively $20 \%, 10 \%$ and $5 \%$. The parameters $\mu$ and $\sigma$ are chosen so that a specific dominance relation holds in the population. For each simulated sample, a series of indicators informing about acceptance or rejection of a given null hypothesis is recorded and then results are reported as averages of these indicators across all simulations. 
Three cases are investigated here. In the first case, I evaluate the size and power of the ISD3 test based on the IF estimator, and I compare it to the behavior of the bootstrap estimator. The first objective is to check the size of the tests by recording how many times over the simulated draws the null is rejected by the data at a nominal size of $5 \%$, knowing that the null is true and cannot be rejected when tested on the population (using one million observations). I consider $F \succcurlyeq_{I S D 3} G$ to be the null hypothesis. Following Barrett and Donald (2009), I assume that each income draw from $F$ is representative of the gross individual-equivalent income from March 1998 CPS data (for the U.S.), where $\mu_{F}=9.85$ and $\sigma_{F}=0.6$. I consider instead that the data drawn from $G$ are generated using $\mu_{G}=9.85$ and $\sigma_{G}=0.7$. The difference between $F$ and $G$ approximates the change of gross individual equivalent income over the 1980s and 1990s. Along with graphical analysis, showing that the generalized Lorenz curves of $F$ and $G$ cross once (hence $F \succcurlyeq_{I S D 2} G$ does not hold), it is possible to conclude in favor of ISD3 by the fact that in the population $\mathrm{E}[F]=\$ 22,697<\$ 24,221=\mathrm{E}[G]$, but $\sqrt{\mathrm{V}[F]}=\$ 14,941<\$ 19,260=\sqrt{\mathrm{V}[G]}$ and $W_{2}(1, F)=\$ 15,238>\$ 15,032=W_{2}(1, G)$ (i.e., the Gini SEF, which is obtained by multiplying the expectation of a distribution by the residual to one of the Gini inequality index). Potentially, $F$ is the distribution yielding higher social welfare for all SEF sufficiently averse to inequality.

The second objective of the Monte Carlo study is to check the power of the ISD tests. This is done by recording the percentage of times in which each test rejects the null when the alternative is true. In this case, I consider two lognormal distributions $F^{\prime}$ and $G^{\prime}$ such that $F^{\prime} \succcurlyeq_{I S D k} G^{\prime}$ for some $k>3$ but not $F^{\prime} \succcurlyeq_{I S D 3} G^{\prime}$. Again, the distributions are parametrized as in Barrett and Donald (2009) to give the most likely guess for the distribution of per-capita nondurable expenditure in the U.S. over the 1990s. Hence, $\mu_{F^{\prime}}=6.37$ and $\sigma_{F^{\prime}}=0.48$ while $\mu_{G^{\prime}}=6.4$ and $\sigma_{G^{\prime}}=0.55$. It is possible to conclude that the generalized Lorenz curves of the two distributions cross once even in this case, and that ISD3 does not hold in the population since $\mathrm{E}\left[F^{\prime}\right]=\$ 655<\$ 700=\mathrm{E}\left[G^{\prime}\right]$ and $\sqrt{\mathrm{V}\left[F^{\prime}\right]}=\$ 333<\$ 416=\sqrt{\mathrm{V}\left[G^{\prime}\right]}$, but $W_{2}\left(1, F^{\prime}\right)=$ 
$\$ 481<\$ 488=W_{2}\left(1, G^{\prime}\right)$.

Detailed results about the size (case 1) and power (case 2) of the tests based on different estimators are reported in Table 1. Concerning the IF estimator (ISD3-IF), the dominance test sizes are generally smaller than 0.10 when inference is made on five abscissae, while there is no clear pattern with respect to the size of the samples. The size of the dominance test increases slightly with the number of abscissae. This is not surprising, given that the test becomes more demanding in terms of comparisons while the number of observations is held fixed, leading to higher likelihood of rejection of the true null hypothesis of ISD3. When the number of abscissae is set to 20, there is evidence of the negative association between size of the sample and size of the test, which evolves from 0.403 when the sample size is small (100 observations) to 0.223 when the sample size is larger (1500 observations). The discriminatory power of the test (case 2) is small when the number of abscissae is set to 5 , with a maximum score of 0.414 . It grows rapidly to acceptable levels (in general larger than 0.7) when the sample size is of 1500 observations. Case 2.a reports the share of cases where the null of equality can be rejected. This share is low (0.095) when the IF estimator is computed for 5 abscissae, indicating that the low power of the dominance test based on the IF estimator is substantially driven by the likelihood that equality of $\Lambda_{3}^{F^{\prime}}$ and $\Lambda_{3}^{G^{\prime}}$ is not rejected by the data. This issue disappears as the sample size or the number of abscissae increases. Case 2.b of the table displays the proportion of cases where rejection of ISD3 null has to be based on simulated p-values, rather than on direct confrontation of the test statistics with the asymptotic boundaries of the rejection regions tabulated in Kodde and Palm (1986). This proportion decreases substantially to less than $9 \%$ as the number of abscissae is set to 20 .

The inference for ISD3 based on the bootstrap estimator (ISD3-BS) yields very similar results as for the IF estimator. The bootstrap estimator shows, nevertheless, somehow larger size and smaller power compared to the IF estimator for any chosen number of abscissae when the sample size is at least as large as 500 observations. For samples of 100 observations, the bootstrap estimator seems to perform better than the 


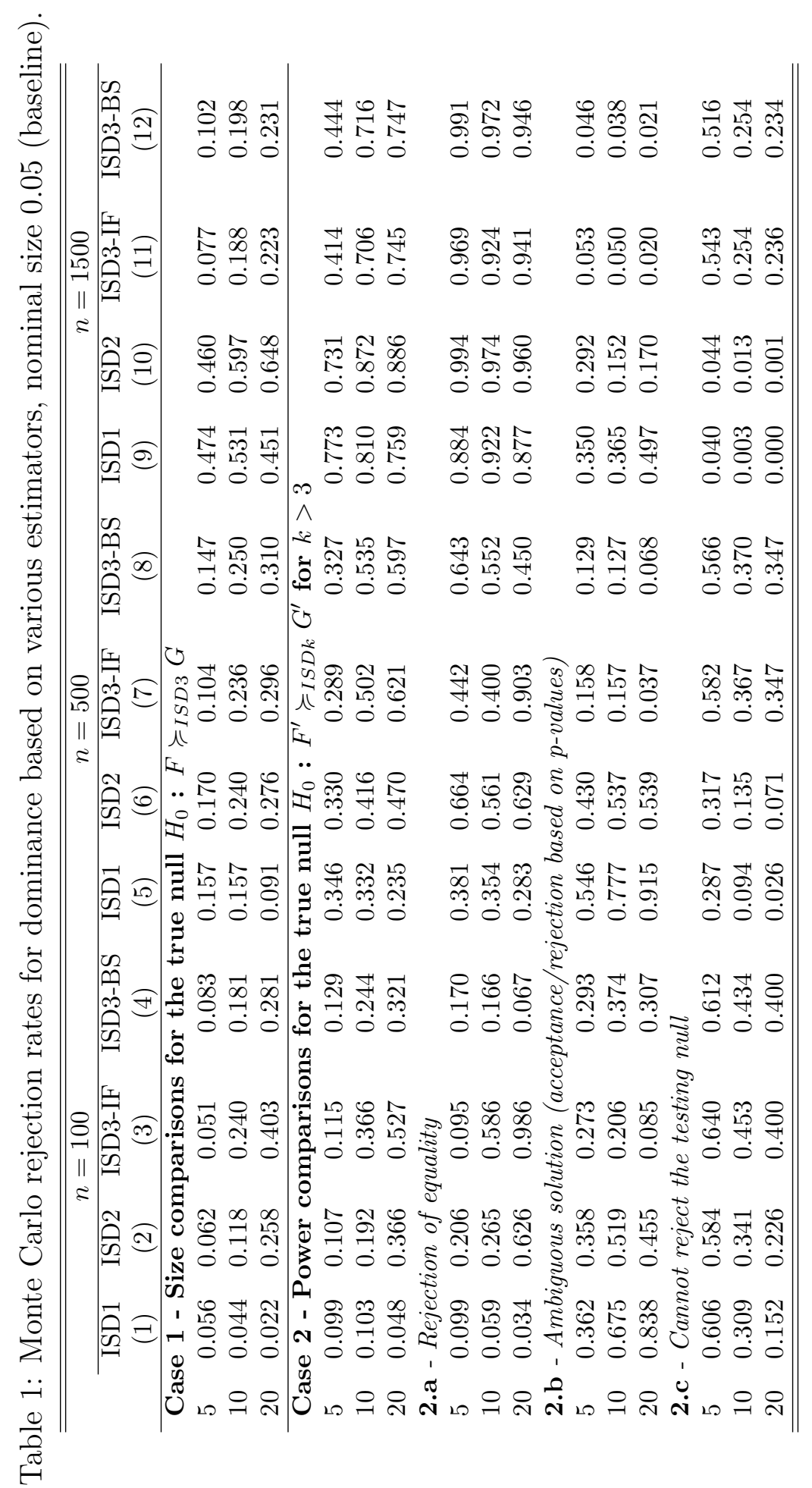

苛

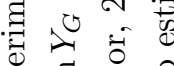
要寻

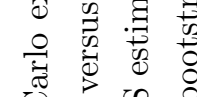
ن की थी

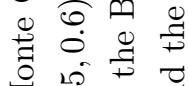

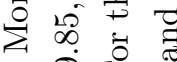
\& 击之的

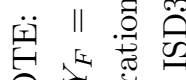

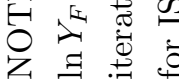


IF estimator in not rejecting the null when the null is true in the population. This comes, however, at the expenses of smaller power.

In the second case, I evaluate the size and power of the ISD3 estimators in the presence of data contamination. To do so, I run Monte Carlo experiments using the same parametrization as above for distributions $F, G$ (for size analysis) and for $F^{\prime}$ and $G^{\prime}$ (for power analysis), while I add some artificial contamination to each simulated draw. Following Cowell and Flachaire (2007), 1\% of observations in each sample have been scaled by 10 , while $1 \%$ have been multiplied by 10 , leading to a total of $2 \%$ contaminated observations. These observations do not necessarily fall on the tails of the distributions. The results of the simulation study are reported in Table 2 and can be interpreted as in Table 1. Once again, the ISD3 tests based on the IF estimator display reasonable patterns of size and power when the sample size is sufficiently large, while the bootstrap procedure has smaller size, but not larger power, when the sample size is of 100 observations. Both size and power of tests for ISD3 based on the IF estimator remain stable after introducing the contamination, indicating that the IF estimator produces robust statistical evaluations of ISD. This is consistent with the finding in Cowell and Flachaire (2007) that the Gini index (upon which the ISD estimator is based) always displays the smaller sensitivity to contamination, even without resorting to appropriate semiparametric methods for modeling the upper and lower tails of the distribution.

In the third and final case, I contrast the size and power of the IF estimator reported in Tables 1 and 2 with tests for second- and third-degree standard stochastic dominance. The stochastic dominance test based on Davidson and Duclos (2000) estimators (denoted DD) consists in evaluating the level of poverty at a given income threshold according to the generalized poverty gap indicator by Foster, Greer and Thorbecke (1984), and to assess how the gaps evolve across the distributions. For stochastic dominance at order two, the evaluation is based on poverty gaps. For stochastic dominance at order three, the square of these gaps is instead adopted. By looking at poverty dominance of $F$ over $G$ at a finite number of poverty thresholds, one 


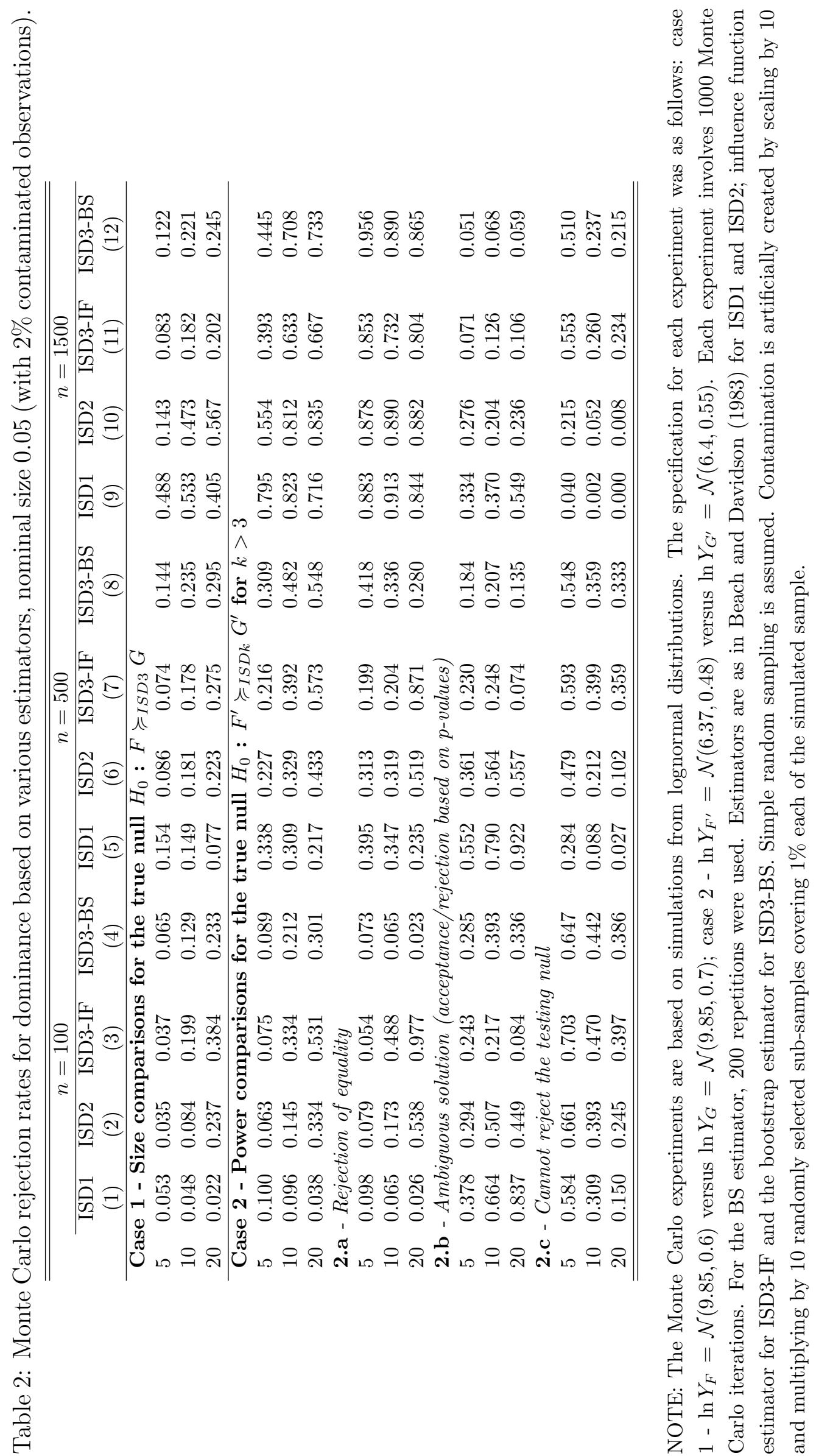




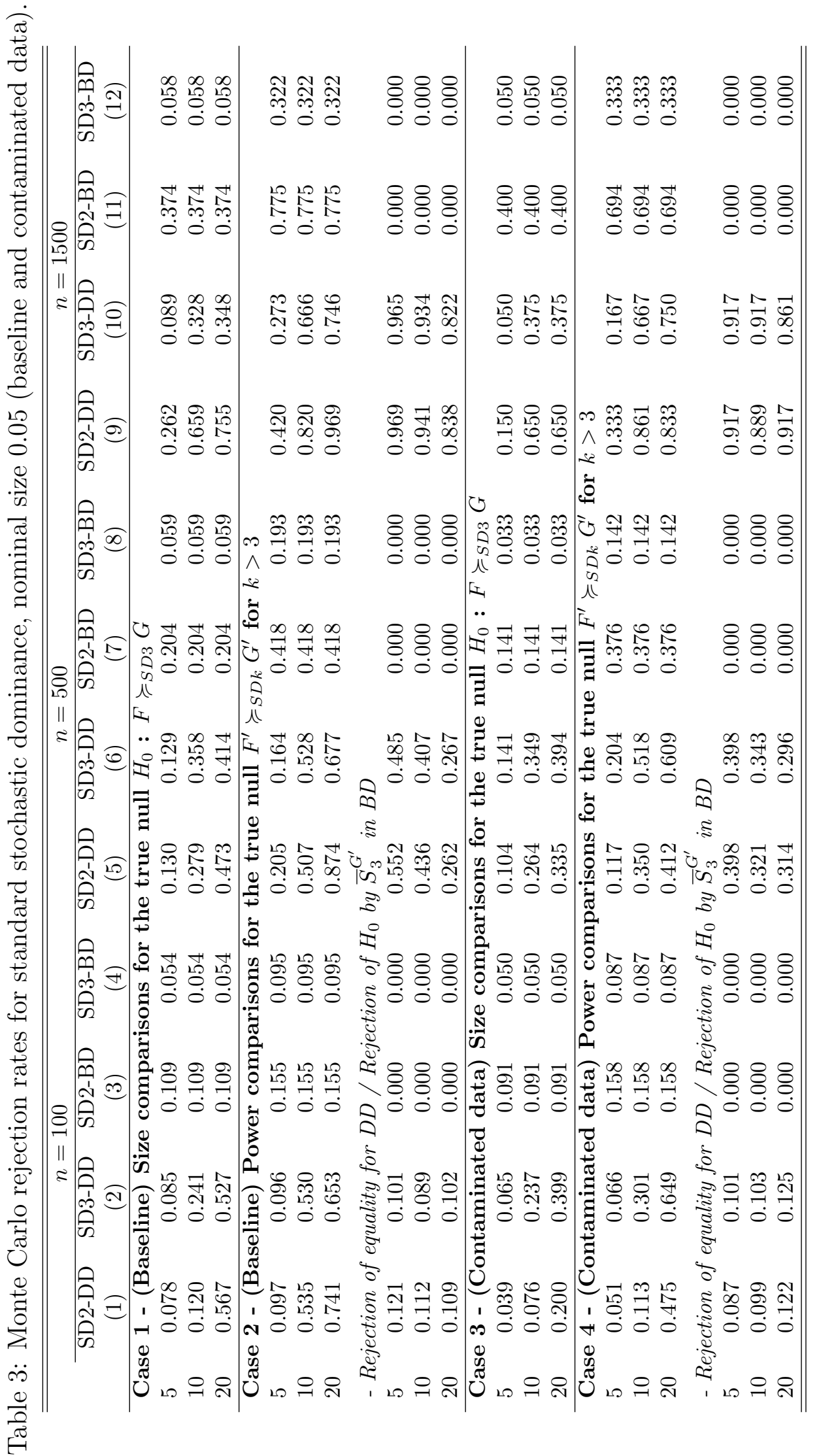

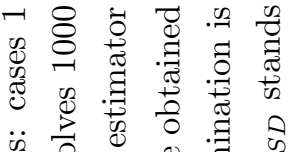

แ

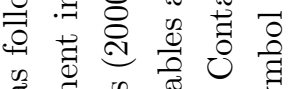

की

हैं

๑ै चี

च.

这官应的

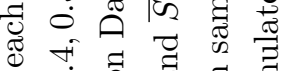

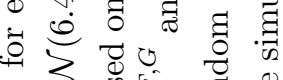

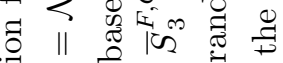

i 800

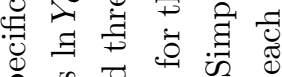

क

क

v

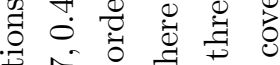

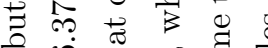

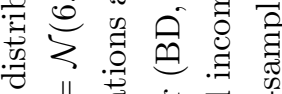

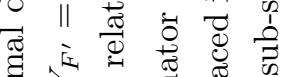

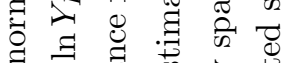
总

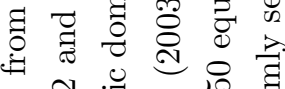

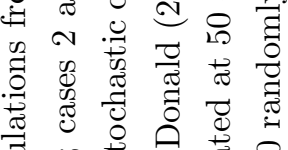

药语

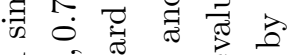

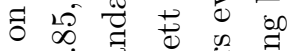

प 0

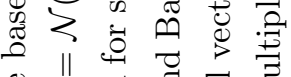

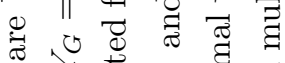

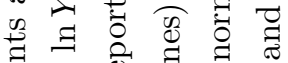

为政

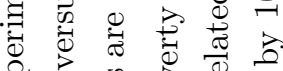

정 울

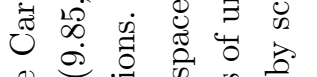

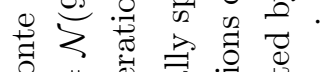

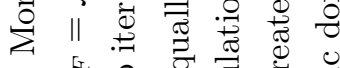

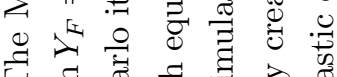

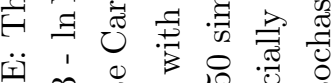

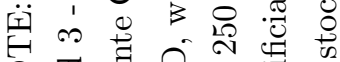

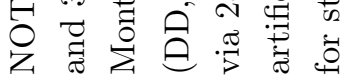


can conclude about standard stochastic dominance using the null hypothesis listed in Section 4 of the published article. I evaluate dominance at 5, 10 and 20 evenly-spaced income thresholds for samples of different sizes obtained from $F$ and $G$ (cases 1 and 3) and from $F^{\prime}$ and $G^{\prime}$ (cases 2 and 4). The stochastic dominance test proposed by Barrett and Donald (2003) (denoted BD) is, instead, a Kolmogorov-Smirnov statistics of the difference between recursive partial integrals of the cdfs taken over the space of income realizations. The statistic is calculated on a fine grid of the income domain (50 thresholds) and BD p-values are simulated. When evaluated in the population (i.e. by drawing a large number of realizations from the distributions), both tests suggest that $F$ third-degree (but not second-degree) stochastic dominates $G$, while $F^{\prime}$ does not stochastic dominate $G^{\prime}$ neither at second- nor at third-degree. Both tests reject the null of equality at conventional levels of significance.

Table 3 reports the Monte Carlo study results for the DD and the BD tests. Focusing on order three stochastic dominance (SD3 in the table) on the baseline case (case 1), it emerges that the size of the SD3-DD estimator is generally larger than the nominal size of 0.05 , and grows with the number of income threshold upon which SD3 is evaluated. The power of the test (case 2) is reasonable only when the number of thresholds is 20 , and somehow increases with the sample size, which also guarantees that the null hypothesis of equality is rejected $90 \%$ of the cases when the sample size is 1500 (but no more than $10 \%$ of the cases when the sample is of 100 observations). The BD estimator (which does not depend upon the number of income thresholds) produces instead tests for SD3 with sizes never exceeding $6 \%$, which is very close to the nominal one. This comes at the expenses of the test power, which reaches the simulated maximum of 0.322 (considerably lower than the power of tests based on the DD and IF estimators) with the largest sample size considered in this study.

Compared to the IF estimator (Table 1), the DD estimator has generally larger size for any number of thresholds and sample size. It also has somehow greater power when the sample size is 500 or below, although the differences between the two estimators levels up once the sample size reaches 1500 units. The baseline case also shows 
that the IF estimator for ISD3 performs comparatively similar to the BD estimator for SD3 when the number of quantiles upon which ISD3 is tested is small. Otherwise, the BD estimator always outperforms the IF estimator in terms of size. The IF estimator, however, outperforms the BD estimator in terms of power in virtually all cases, indicating that the former is more reliable than the latter for the cautious evaluator, who is always willing to discard welfare dominance when it does not hold in the population.

Cases 3 and 4 of Table 3 illustrate the effect of contamination. Size and power of the DD estimator for SD3 are virtually unaltered by contamination, if the number of testing income thresholds is at least 10. This is not surprising given that the poverty gap indicators are robust to contamination (Cowell and Victoria-Feser 2002). When the sample is of at lest 500 observations, the IF estimator is comparably more efficient in not rejecting the true null than the DD test, while the two methods have very similar power scores. One striking difference between the two tests is in terms of the ability they have in correctly rejecting the false null of equality. Both in the baseline and contaminated cases, the DD test seems not to discriminate equality from nondominance when the sample size is smaller than 1500, while testing on 20 thresholds allows to correctly reject on more than $90 \%$ of the times when using the ISD3-IF estimator. Contamination seems to decrease the size of the BD test for SD3, which generally reaches the nominal size and even decreases to $3.3 \%$ when the sample size is of 500 observations. The power of the test, however, never goes beyond 0.33 , which is substantially lower than the power of ISD3-IF estimator.

The Monte Carlo study is based on reliable models of real income and durable consumption distributions in the U.S., already validated in the literature. In the population, the simulation study shows that with these distributions it is never possible to accept generalized Lorenz dominance, the empirical criterion for both ISD2 and standard stochastic dominance at the second order. Higher order dominance becomes relevant. For relatively small samples, the IF estimator for ISD is shown to have the correct size and power in the baseline case, and that these properties remain substantially unaffected by the introduction of artificial contamination in the data. The 
bootstrap estimator displays similar patters but outperforms the IF estimator only for very small samples of 100 observations. From a comparative study with standard stochastic dominance tests, it appears that the IF estimator for ISD3 slightly outperforms the estimator in Davidson and Duclos (2000) when the sample size is large (more than 500 units), although both methods are substantially robust to contaminations. Nonetheless, the IF estimator outperforms the DD estimator in the ability of distinguishing a genuine cross in the curves used to assess ISD3 or SD3 (which implies that the underlying distributions can be ranked at some higher order) from a situations where they are statistically indistinguishable. This is an important issue in evaluation studies, where the impossibility of rejecting the equality null hypothesis might prevent the evaluator from further investigating higher order welfare effects. Finally, in the baseline case the IF estimator produces tests that have generally larger size than the Barrett and Donald (2003) consistent test for stochastic dominance, but that are also considerably superior in terms of power when contamination is an issue.

The figures reported in case 2 of Tables 1 and Table 2, as well as in cases 2 and 4 of Table 3 are useful to assess the power of tests taking generalized Lorenz dominance as the null, when the true null is $\mathrm{ISD} k$ and $\mathrm{SD} k^{\prime}$ for some $k$ and $k^{\prime}$ larger than two. This comparison is informative of the behavior of the tests for ISD2 and for SD2, which have equivalent normative values but rely on substantially different implementation methods. The former is implemented by checking generalized Lorenz dominance at fixed population ranks, the latter at fixed incomes. Confronting power levels in Table 1, columns (2), (6) and (10) with the respective records in Table 3 (baseline case), it emerges that the DD estimator is somehow more powerful than the Beach and Davidson (1983) estimator for ISD2, which in turns dominates the BD method when the number of population proportions' abscissae is above 5. This pattern is substantially preserved when contamination is artificially introduced. Again, we find that in samples of size 500 or less, the ISD2 test is substantially more discriminatory in rejecting the false null of statistical equality of generalize Lorenz curves than the alternative SD2 estimators, although this difference vanishes in samples of larger size. 


\section{References}

Barrett, G. F. and Donald, S. G. (2003). Consistent tests for stochastic dominance, Econometrica 71(1): pp. 71-104.

Barrett, G. F. and Donald, S. G. (2009). Statistical inference with generalized Gini indices of inequality, poverty, and welfare, Journal of Business 8 Economic Statistics 27(1): 1-17.

Beach, C. M. and Davidson, R. (1983). Distribution-free statistical inference with Lorenz curves and income shares, The Review of Economic Studies 50(4): 723735 .

Butler, R. J. and McDonald, J. B. (1989). Using incomplete moments to measure inequality, Journal of Econometrics 42(1): 109 - 119.

Cowell, F. A. and Flachaire, E. (2007). Income distribution and inequality measurement: The problem of extreme values, Journal of Econometrics 141(2): 1044 1072 .

Cowell, F. A. and Victoria-Feser, M.-P. (2002). Welfare rankings in the presence of contaminated data, Econometrica 70(3): 1221-1233.

Davidson, R. and Duclos, J.-Y. (2000). Statistical inference for stochastic dominance and for the measurement of poverty and inequality, Econometrica 68(6): 14351464 .

Deville, J. (1999). Estimation de variance pour des statistiques et des estimateurs complexes: linéarisation et techniques des résidus, Techniques d'enquête 25(2): 219230.

Foster, J., Greer, J. and Thorbecke, E. (1984). A class of decomposable poverty measures, Econometrica 52(3): 761-66.

Kodde, D. A. and Palm, F. C. (1986). Wald criteria for jointly testing equality and inequality restrictions, Econometrica 54(5): pp. 1243-1248. 